\title{
1 The malaria metabolite HMBPP does not trigger erythrocyte terpene production
}

3 Justin J. Miller ${ }^{1}$ and Audrey R. Odom John ${ }^{1,2,3 a}$.

4

$5 \quad{ }^{1}$ Department of Pediatrics, Washington University School of Medicine, St. Louis, MO, USA; ${ }^{2} \mathrm{De}-$

6 partment of Molecular Microbiology, Washington University School of Medicine, St. Louis, MO,

7 USA; ${ }^{3}$ Department of Pediatrics, Children's Hospital of Philadelphia, Perelman School of Medi-

8 cine, University of Pennsylvania, Philadelphia, PA, USA (current institution)

9

10 a Correspondence and requests for materials should be addressed A.R.O.J.

11 (JOHNA3@email.chop.edu)

12 


\section{Abstract}

14 Infection with malarial parasites renders hosts more mosquito attractive than their uninfected,

15 healthy, counterparts. One volatile organic compound, a-pinene, is associated with Plasmodium

16 spp. infection in multiple studies and is a known mosquito attractant. However, how malarial in-

17 fection results in elevated levels of host-associated $\alpha$-pinene remains unclear. One study sug-

18 gests that erythrocyte exposure to the malarial metabolite, (E)-4-hydroxy-3-methyl-but-2-enyl

19 pyrophosphate (HMBPP), results in increased levels of a-pinene. Here, we establish that endog-

20 enous levels of $\alpha$-pinene are present in human erythrocytes, that these levels vary widely by

21 erythrocyte donor, and that $\alpha$-pinene levels are not altered by HMBPP treatment. 
23 Plasmodium falciparum, the primary causative agent of lethal malaria infections, has a two-host

24 life cycle between humans and mosquitoes. Transit between the two hosts is a critical requirement

25 for the parasite lifecycle and represents a substantial population bottleneck ${ }^{1}$. Mosquitoes are more

26 attracted to humans ${ }^{2-5}$, mice $^{6}$, and birds ${ }^{7}$ infected with malaria parasites in comparison to unin-

27 fected, healthy hosts. This observation has led to the hypothesis that Plasmodium species actively

28 manipulate host odor profiles to coordinate transmission to the mosquito. Indeed, changes in the

29 composition of host odor profiles have been observed in humans ${ }^{8-13}$ and mice ${ }^{6}$ infected with ma-

30 laria; however the molecular basis for infection-induced changes in volatile organic compound

31 (VOC) production or release remains unknown.

33 Of particular interest has been the mosquito semiochemical, $\alpha$-pinene, which is found in higher

34 concentrations in the breath of humans with symptomatic Plasmodium infection vs healthy con-

35 trols $^{8}$. Additionally, $\alpha$-pinene has been identified in the headspace above Plasmodium falciparum

36 infected erythrocytes ${ }^{14}$. The VOC $\alpha$-pinene is a member of the large and bioactive class of mole-

37 cules termed terpenes. Terpenes are biosynthesized by a variety of plants, soil and environmental

38 organisms, mammalian commensal and pathogenic microbes, and some insects ${ }^{15-22}$. $\alpha$-pinene is

39 a known component of plant-derived odorant blends that are attractive to the Anopheles spp.

40 mosquitoes that transmit malaria ${ }^{23,24}$. As for other terpenes, biosynthesis of $\alpha$-pinene begins with

41 the 5-carbon isoprenoid precursor, isopentyl pyrophosphate (IPP), which is enzymatically con-

42 densed with a second molecule of IPP by geranyl pyrophosphate synthase (GPPS) to form the

43 10-carbon metabolite, geranyl pyrophosphate (GPP). Subsequent rearrangement and cyclization

44 are catalyzed by a monoterpene synthase (pinene synthase) to yield a-pinene (Fig 1A). 
46 Recently, it was reported that incubation of the microbial metabolite (E)-4-hydroxy-3-methyl-but-

47 2-enyl pyrophosphate (HMBPP) with uninfected human erythrocytes results in increased attrac-

48 tion and feeding behavior of anopheline mosquitoes. Concordantly, an increase in the headspace

49 concentration of $\alpha$-pinene above HMBPP-treated erythrocytes was also reported ${ }^{25}$. HMBPP is a

50 late intermediate in the 2-C-methyl-D-erythritol 4-phosphate (MEP) pathway for synthesis of IPP

51 and downstream isoprenoids (Fig 1A). While eubacteria and apicomplexan parasites, such as

52 Plasmodium spp., utilize the MEP pathway for isoprenoid biosynthesis ${ }^{26}$, humans utilize a distinct

53 and evolutionarily divergent biosynthetic pathway (mevalonate pathway) to synthesize IPP27.

55 The mechanism by which HMBPP exposure of erythrocytes may lead to a-pinene production or 56 release is unclear, but two possibilities may explain these findings. First, HMBPP may serve as 57 an exogenous signal that triggers erythrocytes to release stores of a-pinene which may have 58 accumulated via metabolic, environmental, or dietary routes. A potent activator of human $\mathrm{V}_{\mathrm{\gamma} 9 \mathrm{~V} \delta 2-}$

$59 \mathrm{~T}$ cells ${ }^{28}, \mathrm{HMBPP}$ is recognized as a pathogen-associated molecular pattern (PAMP) through its 60 interaction with butyrophilin receptors ${ }^{29}$, suggesting that HMBPP may serve a signaling role to 61 mediate erythrocyte $\alpha$-pinene release. Alternatively, because HMBPP is itself a precursor to iso62 prenoids and terpenes in bacteria and plants, this metabolite may be directly incorporated into $\alpha-$ 63 pinene in erythrocytes via the pathway illustrated in Fig 1A, or via an as-yet-undescribed alterna-

64 tive enzymatic route. However, human erythrocytes do not express the known biosynthetic ma65 chinery for synthesis of $\alpha$-pinene from HMBPP; mammals lack the MEP pathway and specifically 66 do not express the final enzyme in the pathway, IspH, which converts HMBPP to the immediate 67 a-pinene precursor, IPP. Finally, no erythrocyte monoterpene synthases, nor any proteins with 68 the terpene synthase fold, have yet been described that might mediate the final biocatalysis of 69 GPP to a-pinene. In contrast, humans do express other prenyl diphosphate synthase orthologs, 

we sought to interrogate the possibility of HMBPP-triggered, erythrocyte-produced a-pinene.

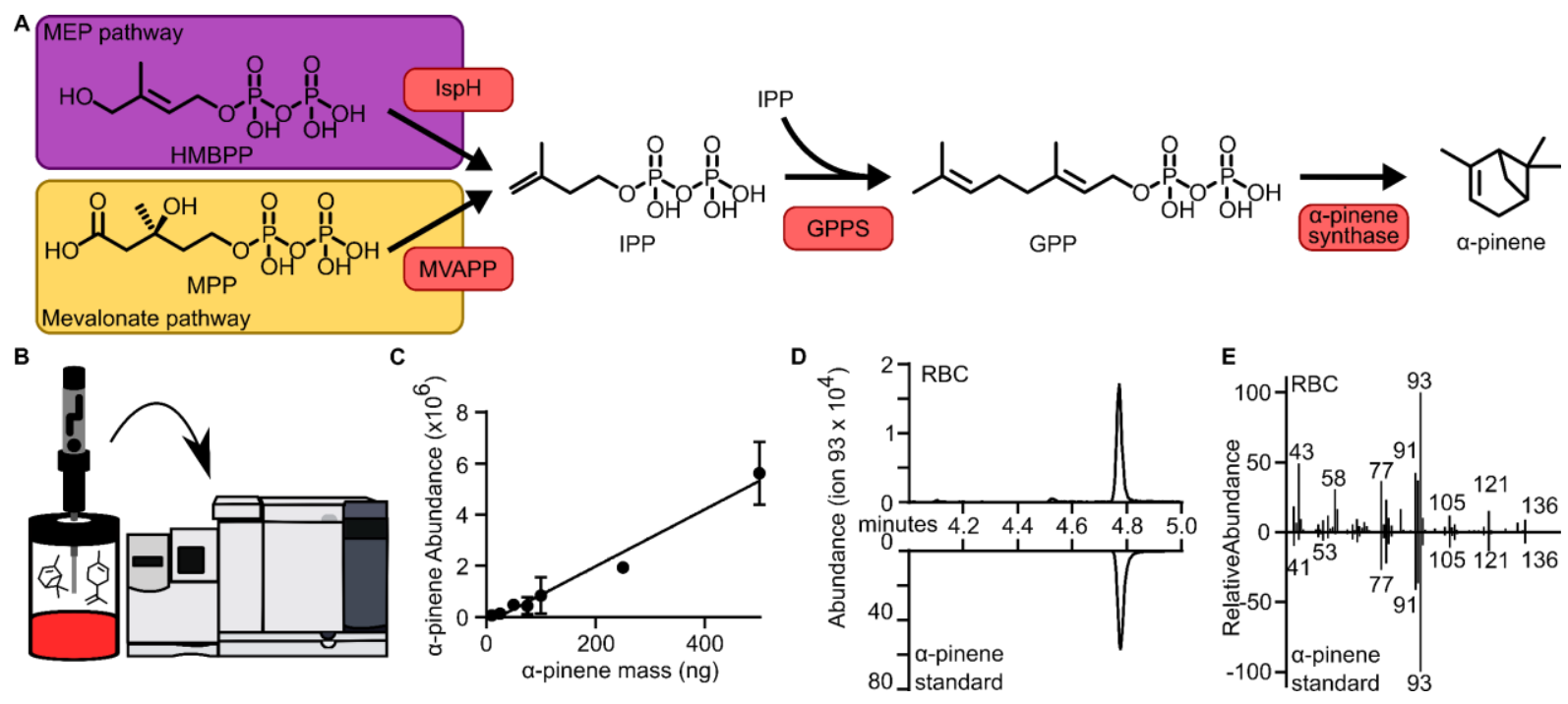

Figure 1. (A) Described metabolic pathways leading to a-pinene. Enzymes highlighted with salmon boxes. (B) Schematic of $\alpha$-pinene detection assay. (C) $\alpha$-pinene standard curve generated using commercial a-pinene. (D) gas chromatography-mass spectroscopy trace of commercial a-pinene (bottom) and erythrocyte headspace (top) for $\alpha$-pinene parent ion, 93. (E) Mass spectra from retention time $4.77 \mathrm{~min}$, the elution time for commercial a-pinene, for erythrocyte headspace (top) and commercial $\alpha$ pinene (bottom).

We established a working method for sampling the volatile organic compounds associated with

74 cultured erythrocytes. Similar to Emami et al., we sealed donated human erythrocytes within a closed, airtight chromatography vial, prewarmed to $38^{\circ} \mathrm{C}$, and performed headspace sampling using solid phase microextraction (SPME) (Fig 1B). Headspace composition was determined using gas chromatography-mass spectrometry. Using a pure commercial $\alpha$-pinene standard, we established the sensitivity and dynamic range of this assay (Fig 1C), yielding a signal-to-noise ratio of 3 and a limit-of-detection of $0.3 \mathrm{ng}$ a-pinene. Accommodating volumes up to $1 \mathrm{~mL}$ of blood, we can detect $\alpha$-pinene blood concentrations as low as $2.2 \mathrm{nmol} / \mathrm{L}$. We next sought to determine

81 whether $\alpha$-pinene was present in the headspace above untreated erythrocytes. Indeed, we confirmed that a-pinene can be detected in the headspace from donor erythrocytes, and both the retention time and mass spectra match that of the pure $\alpha$-pinene standard (Fig 1D,E). 
85 A previous study had indicated that treatment of human erythrocytes with the microbial metabolite

86 HMBPP leads to substantial release of $\alpha$-pinene. To control for batch-to-batch variability in low-

87 level contaminants present in purified HMBPP, we acquired HMBPP from two independent chem-

88 ical suppliers. Headspace sampling from both pure preparations of HMBPP confirmed that neither

89 had contaminating levels of $\alpha$-pinene above our limit-of-detection (Fig 2A). We next treated eryth-

90 rocytes with either HMBPP or water (vehicle control) and quantified headspace a-pinene. Be-

91 cause monoterpenes such as $\alpha$-pinene can diffuse into the ambient air, we pre-aliquoted all blood

92 samples into sealed individual-use aliquots. Treatment of erythrocytes with HMBPP did not result

93 in increased levels of $\alpha$-pinene (Fig 2b), and this finding was not donor-dependent. A previous

94 study also indicated that levels of other monoterpenes ( $\beta$-pinene and limonene), as well as several

95 aldehydes (octanal, nonanal, and decanal), were increased in response to HMBPP treatment. We

96 evaluated for the presence of additional VOCs including these and found all were below the limit-

97 of-detection for our assay. Our studies thus indicate that if erythrocytes can sense HMBPP, this

98 signal is not accompanied by a substantial release of a-pinene. In addition, our studies suggest

99 that erythrocytes do not incorporate exogenous HMBPP for direct de novo synthesis of $\alpha$-pinene.
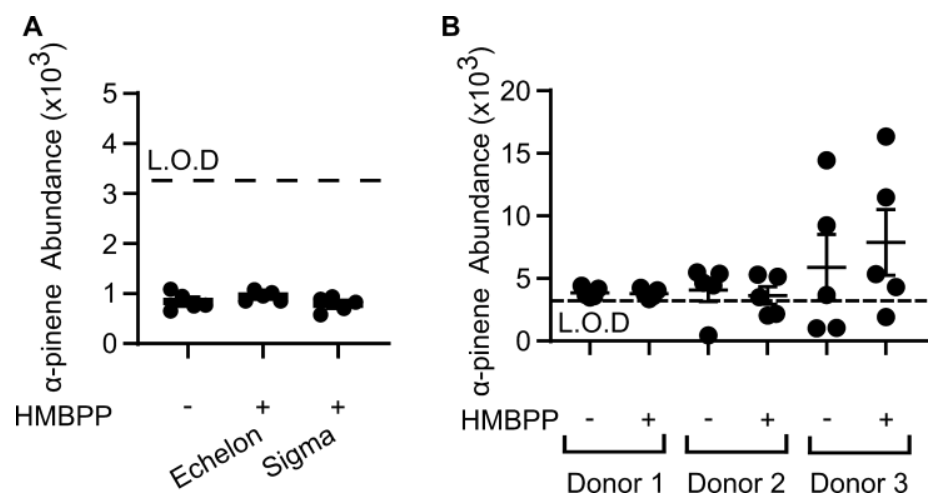

Figure 2. (A) a-pinene abundance in HMBPP from Echelon Biosciences, Sigma Aldrich, or vehicle control (water). (B) Erythrocyte a-pinene abundance following treatment with HMBPP or vehicle control (water). Values are not significantly different by Mann-Whitney $U$ test (Donor $1: p=0.841$, Donor $2: p=0.548$, Donor $3: p=0.420$ ). All assays performed with $n=5$. 


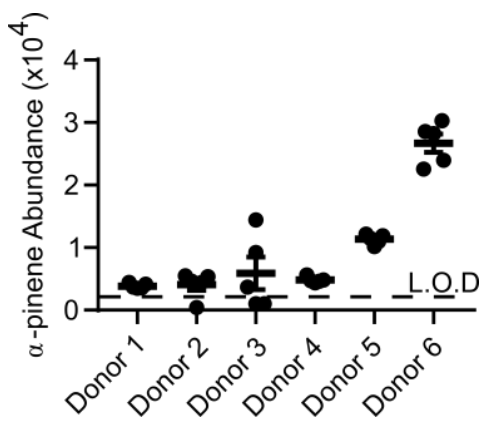

Figure 3. $\alpha$-pinene abundance in the headspace of untreated human erythrocytes, $n=5$.

101 In the course of the above experiments, we noted substantial donor-to-donor variability in the 102 endogenous levels of $\alpha$-pinene present in a given erythrocyte culture. We therefore secured eryth103 rocytes from an additional 3 independent, unrelated donors and quantified a-pinene levels as 104 before. We find that $\alpha$-pinene levels are strongly dependent on donor identity and range widely 105 among our 6 donors (Fig 3). We find that blood $\alpha$-pinene concentrations range from $0.37 \mathrm{ng} / \mathrm{mL}$ $1062.57 \mathrm{ng} / \mathrm{mL}$ (mean and standard deviation, $0.91+/-0.84 \mathrm{ng} / \mathrm{mL}$ ). While biosynthesis of $\alpha$-pinene 107 has not been documented in humans, $\alpha$-pinene is a volatile component of several common dietary 108 plants, suggesting that one explanation for the variability in $\alpha$-pinene levels is due to the variability 109 in diet of individual donors. Alternatively, a-pinene may be synthesized by members of the human 110 microbiome that may contribute to endogenous $\alpha$-pinene levels.

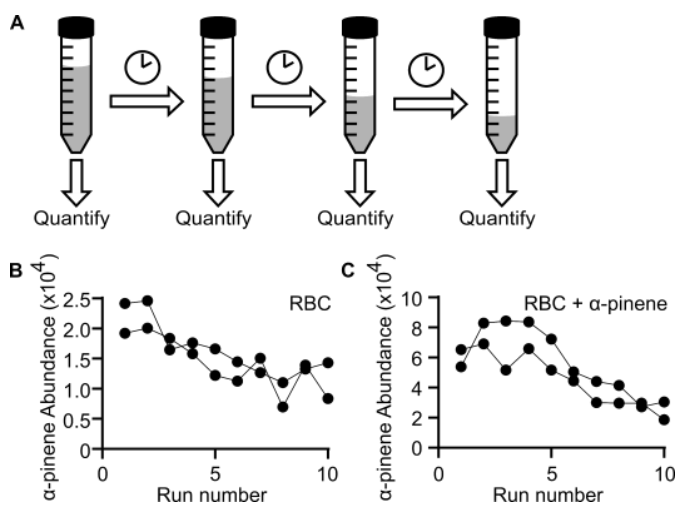

Figure 4. (A) Schematic of repeated sampling mechanism. Time between each sampling is one hour. $(B, C)$ Headspace concentration of $\alpha$-pinene over untreated human erythrocytes (B) or erythrocytes supplemented with $10 \mathrm{ng} / \mathrm{mL}$ commercial $\alpha$-pinene as a function of GC-MS run. 
112 To reconcile our findings with previous studies that had reported HMBPP-induced $\alpha$-pinene re-

113 lease, we hypothesized that the previously described variability may be due to a loss of the volatile

114 a-pinene through diffusion, following repeated sampling of the same sample over time. To test

115 this hypothesis, we filled a single air-tight sample tube with erythrocytes from a single donor. At

$116 \mathrm{t}=0$, we removed $1 \mathrm{~mL}$ of erythrocytes from the conical tube and measured the headspace con-

117 centration of $\alpha$-pinene according to our previous assay. We left the remainder of the sample

118 sealed (Fig 4A). We repeated this process for a total of 10 runs, allowing the tube of erythrocytes

119 to reequilibrate for one hour between sampling. We find that $\alpha$-pinene levels decrease by $25-60 \%$

120 as a result of repeated sampling (Fig 4B). As expected given its vapor pressure $(4.75 \mathrm{~mm} \mathrm{Hg} \mathrm{at}$

$12125^{\circ} \mathrm{C}$ ), a-pinene is in a vapor-liquid equilibrium ${ }^{30}$. Each time our pooled erythrocytes are un-

122 capped and sampled, vaporous a-pinene diffuses away, and a new vapor-liquid equilibrium is

123 established. The total concentration of liquid a-pinene is thus depleted over time, thereby resulting

124 in a reduced pool of $\alpha$-pinene in each subsequent sampling. To confirm that this is not unique to

125 a-pinene naturally absorbed within erythrocytes, we supplemented erythrocytes with $10 \mathrm{ng} / \mathrm{mL}$

126 (73.4 nM) $\alpha$-pinene and found that $\alpha$-pinene levels drop by $66-80 \%$ over the course of repeated 127 sampling (Fig 4C).

129 While run-to-run variability and the run-order effect is a commonly observed problem for mass 130 spectrometry, our results highlight an additional precaution that needs to be taken when sampling 131 biologically generated volatiles. All samples should be aliquoted and sealed in an air-tight con132 tainer prior to the start of the experiment, as repeated sampling from the same container will result 133 in artificially decreased volatile concentration over time. Investigators should continue to control 134 for run-order effects by randomizing the order in which samples are run. 
136 While mounting evidence suggests that Plasmodium infection alters host odor profiles and results

137 in increased mosquito attraction, the mechanism by which this occurs remains unclear. One class

138 of molecules, terpenes, notably $\alpha$-pinene, has been repeatedly highlighted for being both mos-

139 quito attractive and enriched during Plasmodium infection. The metabolic origin of terpenes during

140 Plasmodium infection remains unclear as mammals do not express orthologs of the terpene syn-

141 thases required for terpene production. Here, we establish that endogenous levels of $\alpha$-pinene

142 are present in human erythrocytes. While a-pinene levels from erythrocytes from a single donor

143 sample are highly reproducible, a-pinene levels vary widely by erythrocyte donor. While the

144 source of erythrocyte $\alpha$-pinene remains enigmatic, it is possible that $\alpha$-pinene may be dietary in

145 origin, explaining the donor-to-donor variability that we observe.

147 While HMBPP-mediated $\alpha$-pinene release has been previously reported ${ }^{25}$, in contrast we do not

148 find evidence that the headspace of HMBPP-treated erythrocytes contains increased levels of $\alpha-$

149 pinene. HMBPP-treated erythrocytes also appear more mosquito attractive than untreated eryth-

150 rocytes ${ }^{25}$. Human erythrocytes bind several chemokines ${ }^{31,32}$ and human $\mathrm{V} 9 \mathrm{~V} \delta 2-\mathrm{T}$ cells actively

151 respond to $\mathrm{HMBPP}^{28}$, raising the possibility that $\mathrm{HMBPP}$ exposure of erythrocytes may result in

152 other properties that increase mosquito attraction, independent of $\alpha$-pinene release. $\mathrm{CO}_{2}$ emission

153 from erythrocytes has also been reported to be elevated upon $\mathrm{HMBPP}$ exposure. As $\mathrm{CO}_{2}$ is also

154 a mosquito semiochemical ${ }^{33,34}$, elevated $\mathrm{CO}_{2}$ levels could be responsible for mosquito attraction

155 to HMBPP-treated erythrocytes. However, supplementation of $5 \mathrm{ppm} \mathrm{CO}_{2}$ to untreated erythro-

156 cytes was not sufficient to sway mosquitoes from HMBPP-treated erythrocytes. 
158 Subsequent experiments are needed to identify the origin of Plasmodium infection-associated

159 volatiles. Infection of germ-free animal models may be valuable in discerning volatiles that arise

160 from microbiome vs. host or Plasmodium parasite metabolism. Identification of either human or

161 malarial terpene synthases or metabolic labeling studies are required to understand the origin of

162 Plasmodium infection-associated terpenes. Carefully controlled dietary recall studies are neces-

163 sary to understand whether erythrocyte endogenous a-pinene is biosynthesized by humans or 164 human microbiome members.

165

166 


\section{Materials and Methods}

168 Materials and reagents: (E)-4-hydroxy-3-methyl-but-2-enyl pyrophosphate, HMBPP, was purchased from both Sigma Aldrich and Echelon Biosciences Incorporated (Salt Lake City, Utah, USA), resuspended at $4 \mathrm{mM}$ in highly purified water, and stored at $-80^{\circ} \mathrm{C}$. Human erythrocytes (types A, B, and O, leukocyte reduced and irradiated) were obtained from the St. Louis Children's Hospital Blood Bank (St. Louis, Missouri, USA).

Volatile collection and GC-MS analysis: Erythrocytes were washed 3 times with an equal volume of RPMI-1640 media (Sigma-Aldrich, SKU R4130) supplemented with: 27 mM sodium bicarbonate, $11 \mathrm{mM}$ glucose, $5 \mathrm{mM}$ HEPES, $1 \mathrm{mM}$ sodium pyruvate, $0.37 \mathrm{mM}$ hypoxanthine, $0.01 \mathrm{mM}$ thymidine, $10 \mu \mathrm{g} / \mathrm{mL}$ gentamycin, and 0.5\% albumax (Thermo Fisher Scientific, 11020039) and

177 stored at $50 \%$ hematocrit at $4^{\circ} \mathrm{C}$. When testing responses of erythrocytes to HMBPP and water,

178 erythrocytes were stored as $1.4 \mathrm{~mL}$ aliquots in individual $1.5 \mathrm{~mL}$ microfuge tubes (Thermo Fisher 179 Scientific, 05-408-129), wrapped in parafilm (Sigma-Aldrick, SKU P7793) and stored at $4{ }^{\circ} \mathrm{C}$. Prior 180 to sampling, $1 \mathrm{~mL} 50 \%$ erythrocytes were transferred to $4 \mathrm{~mL}$ glass autosampler tubes (Thermo 181 Fisher Scientific, 03-391-19), closed with screw caps with septa (Thermo Fisher Scientific 03-391182 21), and equilibrated at $38{ }^{\circ} \mathrm{C}$ for 15 minutes. Following equilibration, $2.5 \mu \mathrm{L}$ of $4 \mathrm{mM}$ HMBPP 183 (final concentration $10 \mu \mathrm{M}$ ) or purified water were added to the erythrocytes, caps were closed, and parafilm was used to seal the vial. Volatiles were immediately collected from the headspace using solid phase micro-extraction ( $n=5$, randomized order for each sample). Directly before sampling, the Divinylbenzene/Caboxen/Polydimethylsiloxane fiber (Sigma-Aldrich, SKU 57348) fiber

187 was conditioned for 30 minutes at $225^{\circ} \mathrm{C}$ in the inlet of an Agilent $7890 \mathrm{~A}$ gas chromatographer. Headspace sampling occurred over 30 minutes with temperatures maintained at $38{ }^{\circ} \mathrm{C}$. The volatiles were then desorbed onto the injector of the Agilent 7890A gas chromatographer with an Agilent HP-5MS column (30m, 0.25-mm inner diameter, $0.25-\mu \mathrm{m}$ film thickness) and interfaced with an Agilent 5975C mass spectrometer. The oven program followed a linear temperature 
192 gradient, with an initial temperature of $60^{\circ} \mathrm{C}$ (held for 2 minutes), a ramp of $10{ }^{\circ} \mathrm{C} / \mathrm{min}$ until $225^{\circ} \mathrm{C}$,

193 and a final hold for 5 minutes at $225^{\circ} \mathrm{C}$. Helium was used as the carrier gas with a constant flow

194 of $1 \mathrm{~mL} / \mathrm{min}(25.6 \mathrm{~cm} / \mathrm{sec})$. The ion source temperature, electron energy, and emission current

195 were set at $230{ }^{\circ} \mathrm{C}, 70 \mathrm{eV}$, and $300 \mu \mathrm{A}$ respectively. $\alpha$-pinene was identified based on retention

196 time of an analytical standard (Sigma-Aldrich, SKU 80605), and abundance was quantified using

197 the area under the curve of extracted ion 93. Integration was performed in Agilent MassHunter

198 (Version B.05.00 Build 5.0.519.0) using the Agile integrator. To measure the background contam-

199 ination of HMBPP with $\alpha$-pinene $2.5 \mu \mathrm{L}$ of $4 \mathrm{mM} \mathrm{HMBPP}$ (final concentration $10 \mu \mathrm{M}$ ) was added

200 to $1 \mathrm{~mL}$ of erythrocyte storage media. $\alpha$-pinene standard curve was generated through the addi-

201 tion of $2.5 \mu \mathrm{L}$ of commercial $\alpha$-pinene diluted in hexanes to autosampler tubes containing $1 \mathrm{~mL}$

202 pure water and sampled as with erythrocyte treatments. The limit of detection was defined as 3

203 times the area under the curve (ion 93) at the retention time for commercial $\alpha$-pinene in negative

204 controls containing only water and sampled as with erythrocytes.

206 Measuring $\alpha$-pinene time-dependent concentration: To measure $\alpha$-pinene loss over time, 14

$207 \mathrm{~mL}$ of washed erythrocytes were placed in a $15 \mathrm{~mL}$ conical (Sigma-Aldrich, SKU CLS430791)

208 and stored at $4^{\circ} \mathrm{C}$. For some experiments, analytical $\alpha$-pinene, diluted in water, was added to a

209 final concentration of $10 \mathrm{ng} / \mathrm{mL}$ in erythrocytes at the time of aliquoting. During the experiment,

210 erythrocytes were stored, capped, on ice and iteratively sampled from. Volatile collection and GC-

211 MS analysis proceeded as above. 


\section{References}

214 1. Alavi, Y. et al. The dynamics of interactions between Plasmodium and the mosquito: a 215 study of the infectivity of Plasmodium berghei and Plasmodium gallinaceum, and their 216 transmission by Anopheles stephensi, Anopheles gambiae and <i>Aedes aegypt. Int. J. $217 \quad$ Parasitol. 33, 933-43 (2003).

218 2. Koella, J. C., Sørensen, F. L. \& Anderson, R. A. The malaria parasite, Plasmodium 219 falciparum, increases the frequency of multiple feeding of its mosquito vector, Anopheles 220 gambiae. Proc. Biol. Sci. 265, 763-8 (1998).

221 3. Lacroix, R., Mukabana, W. R., Gouagna, L. C. \& Koella, J. C. Malaria infection increases attractiveness of humans to mosquitoes. PLoS Biol. 3, 1590-1593 (2005).

223 4. Busula, A. O. et al. Gametocytemia and attractiveness of Plasmodium falciparuminfected Kenyan children to Anopheles gambiae mosquitoes. J. Infect. Dis. 216, 291-295 (2017).

5. Batista, E. P., Costa, E. F. \& Silva, A. A. Anopheles darlingi (Diptera: Culicidae) displays increased attractiveness to infected individuals with Plasmodium vivax gametocytes. Parasit. Vectors 7, 251 (2014).

229 6. De Moraes, C. M. et al. Malaria-induced changes in host odors enhance mosquito attraction. Proc. Natl. Acad. Sci. U. S. A. 111, 11079-84 (2014).

231 7. Cornet, S., Nicot, A., Rivero, A. \& Gandon, S. Malaria infection increases bird attractiveness to uninfected mosquitoes. Ecol. Lett. 16, 323-329 (2013).

233 8. Schaber, C. L. et al. Breathprinting reveals malaria-associated biomarkers and mosquito 234 attractants. J. Infect. Dis. 217, 1553-1560 (2018).

235 9. Berna, A. Z. et al. Diurnal variation in expired breath volatiles in malaria-infected and 
healthy volunteers. J. Breath Res. 12, 46014 (2018).

237 10. De Moraes, C. M. et al. Volatile biomarkers of symptomatic and asymptomatic malaria 238 infection in humans. Proc. Natl. Acad. Sci. 115, 5780-5785 (2018).

239 11. Berna, A. Z. et al. Analysis of breath specimens for biomarkers of Plasmodium falciparum 240 infection. J. Infect. Dis. 212, 1120-1128 (2015).

241 12. Robinson, A. et al. Plasmodium-associated changes in human odor attract mosquitoes. $242 \quad$ Proc. Natl. Acad. Sci. U. S. A. 115, E4209-E4218 (2018).

243 13. de Boer, J. G. et al. Odours of Plasmodium falciparum-infected participants influence mosquito-host interactions. Sci. Rep. 7, 9283 (2017).

245 14. Kelly, M. et al. Malaria parasites produce volatile mosquito attractants. mBio 6, 1-6 (2015).

247 15. Stotzky, G. \& Schenck, S. Volatile organic compounds and microorganisms. CRC Crit. 248 Rev. Microbiol. 4, 333-82 (1976).

249 16. Cane, D. E. \& Ikeda, H. Exploration and mining of the bacterial terpenome. Acc. Chem. Res. 45, 463-72 (2012).

251 17. Yamada, Y. et al. Terpene synthases are widely distributed in bacteria. Proc. Natl. Acad. Sci. 112, 857-862 (2015).

253 18. Ditengou, F. A. et al. Volatile signalling by sesquiterpenes from ectomycorrhizal fungi reprogrammes root architecture. Nat. Commun. 6, 6279 (2015).

255 19. Mann, F. M. \& Peters, R. J. Isotuberculosinol: the unusual case of an immunomodulatory 256 diterpenoid from Mycobacterium tuberculosis. Medchemcomm 3, 899-904 (2012).

257 20. Beran, F. et al. Novel family of terpene synthases evolved from trans-isoprenyl 
diphosphate synthases in a flea beetle. Proc. Natl. Acad. Sci. 113, 2922-2927 (2016).

259 21. Gilg, A. B., Tittiger, C. \& Blomquist, G. J. Unique animal prenyltransferase with monoterpene synthase activity. Naturwissenschaften 96, 731-735 (2009).

261 22. Lancaster, J. et al. De novo formation of an aggregation pheromone precursor by an isoprenyl diphosphate synthase-related terpene synthase in the harlequin bug. Proc. Natl. Acad. Sci. 115, E8634-E8641 (2018).

23. Wondwosen, B. et al. Sweet attraction: sugarcane pollen-associated volatiles attract gravid Anopheles arabiensis. Malar. J. 17, 90 (2018).

24. Wondwosen, B. et al. Rice volatiles lure gravid malaria mosquitoes, Anopheles arabiensis. Sci. Rep. 6, 37930 (2016).

25. Emami, S. N. et al. A key malaria metabolite modulates vector blood seeking, feeding, and susceptibility to infection. 4563, 1-9 (2017).

$27026 . \quad$ Lange, B. M., Rujan, T., Martin, W. \& Croteau, R. Isoprenoid biosynthesis: the evolution of two ancient and distinct pathways across genomes. Proc. Natl. Acad. Sci. U. S. A. 97,

273 27. Goldstein, J. L. \& Brown, M. S. Regulation of the mevalonate pathway. Nature 343, 425$27430(1990)$.

275 28. Eberl, M. et al. Microbial isoprenoid biosynthesis and human yठ T cell activation. FEBS $276 \quad$ Lett. 544, 4-10 (2003).

277 29. Rigau, M. et al. Butyrophilin 2A1 is essential for phosphoantigen reactivity by үठ T cells. 
Data Compilation. (Washington, DC: Taylor \& Francis, 1989).

281 31. Darbonne, W. C. et al. Red blood cells are a sink for interleukin 8, a leukocyte

282 chemotaxin. J. Clin. Invest. 88, 1362-9 (1991).

283 32. Neote, K., Darbonne, W., Ogez, J., Horuk, R. \& Schall, T. J. Identification of a promiscuous inflammatory peptide receptor on the surface of red blood cells. J. Biol. Chem. 268, 12247-9 (1993).

286 33. Omondi, B. A., Majeed, S. \& Ignell, R. Functional development of carbon dioxide detection in the maxillary palp of Anopheles gambiae. J. Exp. Biol. 218, 2482-2488 (2015).

289 34. Dekker, T. Carbon dioxide instantly sensitizes female yellow fever mosquitoes to human skin odours. J. Exp. Biol. 208, 2963-2972 (2005). 